\title{
La politica y los politicos en Andalucía Occidental durante la Restauración. Resultados de un proyecto de investigación
}

\author{
RAFAEL SANCHEZ MANTERO
}

En el simposio que la Fundación Ortega y Gasset organizó en Gijón en el mes de junio de 1986, presentaba yo un informe en el que se trazaban las lineas fundamentales de un proyecto de investigación sobre la Restauración en Andalucía Occidental, que por aquel entonces daba sus primeros pasos.

Se trataba esencialmente de invertir la línea que hasta entonces se había seguido en la mayor parte de los estudios de historia política que se habían realizado sobre la etapa de la Restauración. Decía yo en Gijón que con los estudios históricos sobre el largo período de la Restauración estaba pasando lo que con frecuencia ocurría con otras etapas y otras cuestiones importantes de la Historia de España: se había comenzado a construir la casa por el tejado. Quería decir con ello que habíamos llegado a conocer antes el armazón político ideado por Cánovas, los mecanismos generales del funcionamiento del turno pacífico, e incluso la evolución de la práctica política global a lo largo de los años en que estuvo vigente la Constitución de 1876, que los detalles de la estructura partidista local; que la composición, procedencia y comportamiento de la clase política de las capitales y pueblos de las distintas provincias españolas y que muchos otros elementos necesarios para darle más consistencia a cualquier tipo de planteamiento general sobre este tema.

Los pioneros de aquellos trabajos -Comellas, Tusell, Varela Ortega...- eran muy conscientes del coste intelectual que sus esfuerzos habían supuesto, pues habían tenido que suplir la carencia de estudios básicos con generalizaciones no siempre respaldadas suficientemente por datos e informaciones puntuales a escala local.

Estaba claro que había que iniciar otros trabajos en dirección contraria, es decir, de abajo a arriba, para reforzar, matizar y, en su caso, 
modificar, los análisis de la realidad política de aquel tiempo. Para impulsar estos trabajos sobre el área geográfica de Andalucia Occidental, inicié una labor desde el entonces Departamento de Historia Moderna y Contemporánea de la Universidad de Sevilla, consistente en acotar una serie de posibles temas para que fuesen desarrollados en forma de tesis de licenciatura o de doctorado. La idea era que estos temas fuesen abordados a medida que jóvenes graduados con interés por la investigación histórica y que ofreciesen las debidas garantías de continuidad en el trabajo, fuesen incorporándose a un equipo cuyo objetivo iba a ser la suma de esfuerzos con vistas a la realización de un proyecto común.

Debo confesar ante todo, las extraordinarias facilidades que para la puesta en marcha de este proyecto, me ha proporcionado la recluta de excelentes estudiantes que, ya durante los últimos años de carrera, me mostraron su interés por integrarse en él para la consecución del grado de Doctor. La calidad académica y también humana de todos y de cada uno de los componentes del grupo de investigación que han ido incorporándose al mencionado proyecto, ha sido, sin lugar a dudas, la clave de los positivos resultados que hoy podemos presentar aquí, aunque sólo sea de forma esquemática.

Entre los interrogantes que era necesario despejar en una investigación de esta índole, estaba naturalmente el de la identificación de la clase política. No puede entenderse cabalmente y en todas sus implicaciones el complejo funcionamiento del sistema de la Restauración sin conocer los elementos que a escala local, provincial o regional, movían los hilos de la maquinaria puesta en marcha por Cánovas. A pesar de la reducida composición de esta clase política, sabemos en realidad muy poco sobre su procedencia, sobre su preciso status social, e incluso sobre sus intereses reales. En este sentido, nos interesa no sólo reunir la mayor cantidad de datos sobré cada uno de los principales componentes de este entramado, y no sólo de los diputados y senadores, sino de los miembros de los comités locales o provinciales de los partidos de dentro y de fuera del sistema.

El análisis de la clase dirigente que sostuvo todo el sistema de la Restauración y la determinación de su perfil socio-económico, resulta esencial para el conocimiento de las estructuras básicas del poder sobre las que se edificaron los modos políticos de la época. Había, por consiguiente, que rebasar el ámbito de la historia puramente politica para adentrarse en el de la historia social, con todas sus ambigüedades e imprecisiones de fronteras. 
Pero además, interesaba clarificar los mecanismos de relación entre los partidos a escala nacional y los comités locales o provinciales de esos mismos partidos, los modos de funcionamiento de los cuadros de la política sevillana, onubense o gaditana, así como el desarrollo de los procesos electorales a lo largo de todos estos años.

En realidad, se trataba de un ambicioso proyecto, pero que al mismo tiempo era completamente asequible, gracias al material humano disponible y a las fuentes documentales y hemerográficas de carácter local que permitian la consulta de un material de primera mano sin necesidad de los no siempre fáciles desplazamientos a los archivos nacionales. En este sentido, hay que hacer mención especial del interés que se ha puesto desde los inicios de estos trabajos en la localización y explotación de los archivos privados.

La importancia de los archivos privados para el estudio de la vida política en España durante la Restauración, ya fue puesta de manifiesto por Javier Tusell en el Coloquio que se celebró en Madrid sobre "Los Archivos para la historia del siglo XX" (1). Algunos de estos archivos de personalidades políticas relevantes de este período han sido ya utilizados sistematicamente, o están siendo ordenados para su consulta por los investigadores. Por citar algunos de los más importantes, los de Antonio Maura, Eduardo Dato o Manuel Giménez Fernández. Sin embargo, todos sospechamos de la existencia de archivos privados, de mayor o menor envergadura, en manos de descendientes de aquellos personajes que cubrieron un papel más o menos relevantes en la política local. Como también sospechamos de la pérdida irremediable de importante documentación personal de estos políticos a causa de la desidia o de la simple ignorancia de sus depositarios.

En nuestro proyecto, además de la utilización de los archivos privados ya conocidos, como los mencionados ya, o el de Burgos y Mazo, ha podido utilizarse ampliamente el archivo de la familia Ybarra. Un importante archivo éste, en el que se conserva toda la documentación de tipo político y económico que generaron los miembros de esta familia, de origen vasco, que se afincó en Sevilla a mediados del siglo XIX y que desempeñó un papel relevante en la vida de la capital andaluza. Igualmente se ha utilizado el archivo carlista, depositado también en Sevilla y controlado por la familia Fal Conde, de fundamental importancia para el estudio del movimiento carlista en

(1) TUSELL, Javier: "Los Archivos para la Historia Política del reinado de Alfonso XII" en Los archivos para la bistoria del siglo XX. Madrid, 1986. 
España, prácticamente desde sus origenes. De un interés más limitado, se han sacado a la luz otros archivos personales, como el de la familia Ordoñez, para el estudio del sistema caciquil en la Sierra de Aracena, o el de la familia López de Carrizosa para el mismo objeto en Jerez. En ocasiones, han podido obtenerse también algunos papeles dispersos por los procedimientos más diversos e incluso, a veces, hasta pintorescos (2).

Los resultados de este interés por descubrir y salvar la mayor cantidad posible de archivos de este tipo han sido ya bastante positivos, pero sobre todo está permitiendo crear un ambiente de seguridad y de confianza en la opinión pública que hará, sin duda, que en un futuro próximo podamos contar con nuevos ofrecimientos por parte de particulares que conserven documentos de aquella época.

El primer trabajo de investigación que se llevó a cabo dentro de este proyecto fue el de Karin Puech Suances, titulado "Política y políticos sevillanos en los inicios de la Restauración.1874-1881". Se trataba de una tesis de licenciatura, defendida en 1980, en la que se analizaba la puesta en marcha en Sevilla y su provincia del sistema político articulado por Cánovas del Castillo. A lo lago de cerca de trecientas páginas, Karin Puech estudiaba de forma minuciosa y densa el proceso de formación de las dos grandes fuerzas políticas, conservadora y liberal, en la capital andaluza y todas las dificultades que hubo que vencer para aglutinar las diversas fracciones que se movian en el estrecho mundo provincial.

Este trabajo, a pesar de su indudable interés y de que ha servido como punto de referencia inevitable para todas las investigaciones que se han llevado a cabo posteriormente sobre el tema, no ha podido ser publicado hasta el momento. Su autora, por otra parte, optó para la realización de su tesis doctoral, por razones que no son del caso exponer aquí, por un tema relacionado con la. Historia económica, que ya ha culminado también con brillantez.

El periodo subsiguiente al tratado por Karin Puech, es decir, el comprendido entre 1881 y 1898, fue acotado para otra tesis de licenciatura que comenzó a realzarse con mucho entusiasmo, pero que fue abandonada cuando ya se había recogido gran parte del material

(2) Una relación de los archivos privados, en la ponencia que presenté en los "V Coloquios de Historia de Andalucía. Andalucía Contemporánea", organizados por el Monte de Piedad y la Caja de Ahorros de Córdoba en mayo de 1990 y que lleva por título Los archivos privados para la Historia Contemporánea de Andalucía y que actualmente está en prensa. 
hemerográfico. El licenciado frustrado, Juan Manuel Molina Lamothe, que hoy forma parte de nuestro cuerpo diplomático, nos dejó sin embargo un artículo sobre las repercusiones del 98 en Sevilla (3).

Aunque centrado en la época posterior de la Dictadura de Primo de Rivera, Leandro Alvarez Rey trazó con acierto en su tesis de licenciatura un esquema general sobre el funcionamiento de la política local en estos años, dentro de este mismo proyecto de investigación. En su trabajo, Alvarez Rey analizó la recomposición de las fuerzas políticas sevillanas entre 1923 y 1930 y el reforzamiento de una nueva agrupación que jugó un papel importante en esta etapa: la Unión Comercial, integrada por empresarios y comerciantes, cuya proyección llegó más allá de la propia Dictadura. Este interesante estudio mereció el premio de la Diputación sevillana en 1986 y fue publicado por la misma institución (4).

El profundo conocimiento que Alvarez Rey adquirió sobre los entresijos de la política sevillana en los últimos momentos de la Restauración, le llevó, por motivos de pura curiosidad intelectual; a prolongar su estudio hasta abarcar toda la etapa de la Segunda República. El proceso de readaptación y de nueva toma de posiciones de la derecha sevillana a partir de la proclamación de la República, sería el objetivo de la tesis de doctorado. Dicha tesis, con el titulo de "La derecha sevillana durante la Segunda República" fue defendida en octubre de 1990 y actualmente se halla en vias de publicación por la Universidad de Sevilla.

'Alvarez Rey ha completado en su trabajo el concienzudo estudio que realizó en su día José Manuel Macarro sobre la izquierda sevillana en la misma etapa (5), de tal manera que hoy podemos contar con un conocimiento muy completo sobre tan crucial periodo de la reciente historia sevillana. Pero además, Alvarez Rey ha incluido en su trabajo un análisis de la evolución de las derechas sevillanas desde comienzos de nuestro siglo, en el que se refiere no sólo a los partidos del sistema, sino a los grupos marginales, a las llamadas "verdaderas derechas", como la Liga Católica, los carlistas, los mauristas y la Unión

(3) MOLINA LAMOTHE, Juan Manuel: "La crisis del 98 en Sevilla: del cierre de tiendas al nacimiento de la Unión Nacional. 1898-1900", en I Congreso de Historia de Andalucia (Siglos XIX y XX), tomo II. Córdoba, 1976.

(4) ALVAREZ REY, Leandro: Sevilla durante la Dictadura de Primo de Rivera (La Unión Patriótica. 1923-1930). Sevilla, 1987.

(5) MACARRO VERA, J.M.: La utopia revolucionaria. Sevilla en la Segunda República. Sevilla, 1985. 
Comercial. Todo ello le ha permitido ofrecer una clara explicación de la postura que estas derechas adoptaron ante las vicisitudes de la política española y realizar una profunda reflexión acerca de las razones que llevaron a muchos sevillanos a apoyar la opción que estas derechas representaban. La inclusión de numerosos cuadros y estadísticas cuidadosamente elaborados enriquecen considerablemente el estudio de Alvarez Rey y permitirán al lector contar con una fuente de datos de inestimable valor (6).

A pesar de que tampoco encaje exactamente dentro del período cronológico de la Restauración, es obligado citar aquí también el minucioso y extenso trabajo de Eloy Arias Castañón sobre los origenes del partido republicano en Sevilla. En realidad, su propósito inicial era el de abordar, dentro del proyecto de investigación sobre "La política y los políticos sevillanos durante la Restauración", el caso del republicanismo. Lo que ocurría era que resultaba necesario analizar necesariamente los precedentes del republicanismo y eso le llevó a adentrarse en la complicadísima vida política de la Sevilla del Sexenio Revolucionario. Salvo el artículo que Carlos Martínez Shaw publicó sobre el cantón sevillano (7), que era en realidad un resúmen de su tesis de licenciatura, no se había llevado a cabo ningún estudio serio sobre el Sexenio en la capital andaluza. Por ello era necesario desbrozar previamente el terreno que podría permitir, posteriormente, iniciar sobre una base sólida el estudio del republicanismo durante la Restauración.

Eloy Arias presentó su Memoria de licenciatura en 1986 (8) después de un intenso trabajo de acopio documental. Su denso estudio, que desbordaba ampliamente los límites que razonablemente se le atribuían a estos trabajos, mereció el premio convocado por el Ayuntamiento de Sevilla para la mejor tesis de licenciatura de aquel año en el distrito . Sin embargo, el elevado número de páginas de este estudio y la imposibilidad, hasta el momento, de que su autor se haya decidido a la penosa tarea de reducción del original, han impedido por ahora su publicación.

(6) Por necesidades editoriales, Alvarez Rey se ha visto obligado a separar este análisis de la evolución de las derechas sevillanas desde principios de siglo del resto de su estudio, para publicarlo en el tomo I de Sevilla en el Siglo XX, de la colección Historia de Sevilla. Sevilla, 1990.

(7) MARTINEZ SHAW, C.: "El cantón sevillano", en Arcbivo Hispalense, 170. Sevilla, 1972.

(8) ARIAS CASTAÑON, E.: Republicanismo y vida politica en Sevilla, 1868-1874. Tesis de Licenciatura inédita. Sevilla, 1986. 
En la actualidad, Eloy Arias ha culminado la fase de recogida del abundante material hemerográfico y documental para la redacción de su tesis de doctorado sobre el tema inicial previsto -"El republicanismo sevillano durante la Restauración"- y es de esperar que en breve plazo de tiempo podamos contar con su interesante estudio sobre la cuestión.

El funcionamiento del partido conservador en Sevilla durante la segunda etapa de la Restauración, es decir, a partir de comienzos de nuestro siglo, está siendo objeto de estudio por parte de María Sierra Alonso. Ella misma ha formulado claramente su objetivo y a sus palabras nos atenemos: "Desde un primer momento nos pareció necesario el afrontar el estudio de la naturaleza del sistema político de la Restauración desde el análisis de la clase dirigente que lo mantuvo y usufructuó: la determinación de la posición concreta de este grupo respecto al resto de la sociedad, y de las consecuencias de ese status socio-económico en la vida política, permitía ofrecer la estructura básica de poder en virtud de la cual se configuraron los modos políticos característicos de la Restauración. El conocimiento de las redes de clientelas, sus patrocinadores, la relación de preeminencia o de subordinación de éstos respecto al poder central, el tipo de lazos de dependencia, etc., explicará en parte la existencia de esas determinadas manifestaciones políticas, como el encasillado o los mecanismos de presión electoral, que, aunque se hacían más patentes en los momentos electorales, se debían a una realidad de fondo que funcionaba cotidianamente.

La opción metodológica expuesta se vio reforzada -sigue diciendo María Sierra- por el hecho de poder acceder a un tipo de documentación que consideramos privilegiada. Se trata del bien conservado archivo de la familia Ybarra, jefes del Partido Conservador y eminentes miembros de la oligarquía económica provincial".

En efecto, la gran suerte de María Sierra ha sido la de poder contar con un rico archivo privado, cuya documentación está constituyendo el núcleo fundamental de la información sobre su tema. Además el archivo Ybarra cuenta con una colección de prensa, formada por periódicos de carácter conservador y cuyos ejemplares son inexistentes en la Hemeroteca Municipal de Sevilla. Hasta su utilización por María Sierra, este archivo sólo había sido explotado parcialmente por Eduardo Ybarra Hidalgo, descendiente de aquellos Ybarra de principios de siglo y depositario actual del repositorio documental (9).

(9) Cfr. YBARRA HIDALGO, E.: Noticias sevillanas de cinco bermanos. Sevilla, 1985. 
La investigación de María Sierra ha dado lugar ya a la realización de un trabajo de doscientas páginas que fue presentado como Memoria de Tercer Ciclo en la Universidad de Sevilla en 1989 (10) y que está en proceso de convertirse en letra impresa, así como a algunas otras publicaciones sobre distintos aspectos de la cuestión (11). Sin embargo, su objetivo final es el de la realización de su tesis doctoral sobre el partido conservador sevillano en su conjunto. Actualmente esta tesis de halla en fase avanzada de elaboración y seguramente podrá ser defendida ante el correspondiente tribunal en el curso del año 1993.

Entre los diversos aspectos que era necesario cubrir también en este proyecto estaba el de estudiar otros partidos de menor relevancia, pero también importantes para mejor entender las claves del funcionamiento de la política de la Restauración en Sevilla. Tal era el caso de la Liga Católica, impulsada y promocionada por el Cardenal Marcelo Spínola desde el arzobispado. Esta iniciativa de movilización de los católicos dio lugar a la creación de El Correo de Andalucía, el periódico más antiguo de los que aún se siguen publicando en Sevilla. En el seno de la Liga se forjaron también importantes políticos sevillanos como Manuel Rojas Marcos, su fundador, y Manuel Giménez Fernández, entre otros.

José Leonardo Ruiz Sánchez se ha encargado del estudio de esta formación política desde su fundación en 1901 hasta 1923. Desgraciadamente, y a pesar de los esfuerzos desplegados, no ha sido posible hasta el momento localizar el archivo de El Correo de Andalucía, que sería de gran importancia para determinar cuestiones tan cruciales como los medios económicos con los que inició su andadura este órgano del partido, la relación del periódico con el arzobispado, la lista de los accionistas, etc. José Leonardo Ruiz ha rastreado, no obstante, todos los archivos sevillanos y otros de fuera de Sevilla, incluido el Archivo Vaticano, para analizar los propósitos y las actividades de la Liga Católica. Fruto de su intenso trabajo ha sido la presentación en 1988 de un interesante estudio como memoria de Tercer Ciclo titulado "Política e Iglesia sevillana durante la Restauración. Los origenes de la Liga Católica", en el que abordó fundamentalmente los aspectos fundacionales y justificativos de este grupo político confesional.

(10) SIERRA ALONSO, M.: Empresarios y politicos en la Sevilla de la Restauración: la Familia Ybarra. Memoria del Tercer Ciclo inédita. Sevilla, 1989.

(11) Cfr. por ejemplo el artículo del mismo título publicado en Espejo. Tiempo $y$ Forma. Revista de la Facultad de Geografia e Historia. UNED. Serie V. 3/1. Madrid, 1990. 
En su estudio, José Leonardo Ruiz analizó la procedencia de los distintos elementos que participaron en la fundación de la Liga: integristas, carlistas y algunos elementos del partido conservador, además de otros católicos sin antecedentes de adscripción partidista alguna. Realizó también un seguimiento de la evolución numérica de los militantes del partido, de tal manera que hoy podemos saber que, al poco tiempo de su fundación, la Liga contaba ya con 2.495 individuos, de los cuales 1943 residían en Sevilla y el resto se repartía entre Huelva ,Cádiz y Córdoba. También estudió la labor de los concejales que fueron elegidos para el Ayuntamiento de Sevilla; así como la del propio Rojas Marcos, diputado a Cortes en 1918 y 1919 (12).

La tesis doctoral que prepara J. Leonardo Ruiz, en la que tiene el propósito de completar este estudio sobre la Liga Católica, podrá ofrecernos, sin duda, una aportación fundamental para el conocimiento del catolicismo social y político en la última etapa de la Restauración y que derivaría más tarde hacia la formación de una democracia cristiana, en la que militaron muchos de los antiguos miembros de esta agrupación.

La culminación de algunos de estos trabajos y la progresión de los otros, permiten ser optimistas en cuanto al cumplimiento de los objetivos iniciales del proyecto para el ámbito sevillano. No obstante, era factible también ampliar el estudio sobre la política y los políticos de la Restauración a otros ámbitos andaluces, aplicando sencillamente los procedimientos y la metodología empleados para Sevilla. Por otra parte, el hecho de que el Departamento de Historia Moderna y Contemporánea de la Universidad de Sevilla impartiese también sus enseñanzas en Huelva, permitía esperar que algún alumno destacado mostrase interés por abordar el estudio de la cuestión en esta provincia. En efecto, en 1989, la recién licenciada en la Facultad de Huelva María Antonia Peña Guerrero se integró en el equipo de investigación para realizar su tesis de doctorado sobre "La vida política en Huelva durante la Restauración", para lo que le fue concedida una beca del Programa de Formación del Personal Investigador del Ministerio de Educación y Ciencia.

El estudio de la élite política en Huelva tendría que partir, aún más que en el caso de Sevilla, de cero, en el sentido de que existía una carencia absoluta de estudios sobre la Huelva de este período. De otro lado, sin embargo, la menor relevancia de la actividad política en esta

(12) RUIZ SANCHEZ, J.L:: "Los católicos sevillanos ante el reinado de Alfonso XIII: entre la tradición y el progreso". Espacio. Tiempo y Forma. Revista de la Facultad de Geografia e Historia. UNED. Serie V. 3/1. Madrid, 1990. 
provincia en el marco general de la política española, podía permitir un enfoque más amplio de la cuestión, de tal forma que no fuese necesario parcelar excesivamente toda la etapa de la Restauración. En un principio se acotaron los dos tramos cronológicos generalmente aceptados para este período: el primero desde 1874 hasta 1898 , y el segundo desde comienzos del siglo XX hasta la implantación de la Segunda República. Manuel Lara Ródenas, también licenciado por la Facultad de Huelva, fue el encargado, en una primera distribución del trabajo, de analizar el desarrollo de la vida política onubense en el siglo XIX. Sin embargo, circunstancias personales le obligaron temporalmente a posponer su estudio.

María Antonia Peña por su parte ha desarrollado con éxito su trabajo sobre la etapa conocida como la de "La crísis de la Restauración", de tal manera que pudo defender ya en diciembre de 1990, ante el correspondiente tribunal, su Memoria de Tercer Ciclo sobre "Política y políticos en Huelva en los inicios del siglo XX". En ella analizaba el comportamiento de las principales personalidades onubenses que movían el engranaje político en la provincia, así como la evolución de los partidos del turno durante los primeros años de nuestra centuria.

Fruto de su labor investigadora en este terreno, han sido también diversos artículos y comunicaciones en reuniones científicas sobre el tema de la política de la Restauración (13) que ponen de manifiesto su excelente nivel de dedicación así como su dominio de las fuentes disponibles en Huelva y fuera de Huelva. Todo ello tiene como objetivo final la realización de la tesis de doctorado, que a buen seguro podrá ser defendida en el curso del año 1992.

Por último, es necesario mencionar también el intento que se está llevando a cabo de analizar la vida política en Jerez durante esta etapa. La encargada de llevar a buen puerto esta tarea es la licenciada Inmaculada Morales López, que se halla todavía en la fase inicial de localizar las fuentes disponibles para ello. En su haber hay que contabilizar ya la localización de lo que al parecer es un interesante archivo privado, perteneciente a la familia López de Carrizosa -ya mencionado anteriormente-, uno de cuyos miembros desempeñó un importante papel en la política conservadora jerezana de comienzos de siglo.

(13) Cfr. PEÑA GUERRERO, M.A.: "La élite política en Huelva ante las elecciones de 1923". Espacio. Tiempo y Forma. Revista de la Facultad de Geografia e Historia. UNED. Serie V. 3. Madrid, 1990; y PEÑA GUERRERO, M.A.; LARA RODENAS, M. y DOMINGUEZ DOMINGUEZ, M.: "La crisis de 1917 en Huelva". Huelva en su Historia, 3. Huelva, 1990. 
Pues bien, todos estos trabajos se complementan adecuadamente con otras investigaciones que, si bien no tienen su objetivo enfocado al ámbito de lo político, si contribuyen a enriquecer los conocimientos sobre la etapa que nos ocupa, y por tanto a facilitar puntos de apoyo a quienes se dedican a esta tarea específica. A este respecto hay que citar la tesis de licenciatura de Eloisa Baena Luque sobre "El trabajo de la mujer en Sevilla a comienzos del siglo XX" (14), o las varias publicaciones de María Angeles González sobre el movimiento obrero en Sevilla y que son avances de su tesis que dirige el profesor Macarro Vera (15).

En el terreno académico resulta casi siempre muy difícil cumplir los plazos propuestos en un principio, cuando se trata de trabajos colectivos cuya realización está sujeta siempre a circunstancias de tipo personal, o incluso académico-administrativas, que hay que atender aunque sea a costa de relentizar la investigación que se tiene entre manos. Aún así, creo que en este caso los resultados obtenidos hasta el momento por los integrantes de este equipo de personas son dignos de tenerse en cuenta y, en su conjunto, representan una significativa muestra de lo que puede rendir un grupo de jóvenes investigadores con vocación, capacidad de trabajo y excelente nivel científico.

El proyecto de investigación sobre "La política y los políticos en Andalucía Occidental durante la Restauración" incluía también la realización de un banco de datos sobre los individuos que desempeñaron algún papel en la vida política de estos años. El propósito de este capítulo del proyecto es el de incluir en él, no sólo a los personajes más relevantes, como los diputados (16) y senadores, o los jefes locales de cada una de las agrupaciones existentes durante este largo período de la historia contemporánea andaluza, sino a todos aquellos que ocuparon alguna vez un puesto de concejal, directivo de un comité local, o algún cargo público entre 1874 y 1931 . En total más de cinco mil personas, sobre las que hay que recabar datos sobre su trayectoria personal, sobre su actividad política, sobre su situación económica y sobre su perfil social.

(14) BAENA LUQUE, E.: El trabajo de la mujer en la Fábrica de Tabacos de Sevilla a comienzos del siglo XX. Memoria de Tercer Ciclo inédita. Sevilla, 1989.

(15) Véase por ejemplo, GONZALEZ, M.A.: Lucba obrera en Sevilla. Conflictividad social 1900-1917. Barcelona, 1988.

(16) Sobre los diputados sevillanos, véase mi artículo "Los diputados sevillanos durante la Restauración", Archivo Hispalense, 220, Sevilla, 1989, en el que se incluyen algunos datos muy esquemáticos, pero significativos, sobre cada uno de ellos. 
En realidad, muchos de estos personajes aparecen ya identificados en los trabajos de investigación que acabo de mencionar, de tal manera que basta con extraer sus datos para incluirlos en una ficha especialmente confeccionada para ello y clasificarla convenientemente en un fichero general, a partir del cual se puedan realizar consideraciones de carácter general sobre este numeroso colectivo. Esta tarea se vería facilitada con la informatización de esos datos, ya que así su tratamiento ofrecería muchas más posibilidades y una mayor rapidez en la extracción de conclusiones. A ese objeto, el Centro de Cálculo de la Universidad de Sevilla confeccionó un complejo programa de acuerdo con las necesidades planteadas para este objetivo del Proyecto de Investigación. Esa misma complejidad, y otras dificultades de infraestructura y de personal, han retrasado hasta el momento la puesta en marcha de este programa. No obstante, la inclusión de todo el Proyecto en el Plan Andaluz de Investigación (PAI) en el año 1991 facilitará de una manera definitiva la realización del banco de datos, así como la ampliación de los objetivos iniciales.

A la vista de los resultados aquí expuestos, cabría hacer algunas consideraciones en torno a las pautas que han marcado a lo largo de estos años, la realización de estos trabajos. En realidad, lo primero que habría que preguntarse es qué es lo que sabemos hoy sobre la cuestión, que no supiésemos cuando comenzamos a recorrer este largo camino. Pues bien, hay que decir que la información y el conocimiento que hoy tenemos sobre el funcionamiento de los mecanismos del sistema de la Restauración en Sevilla y en las otras provincias estudiadas, nos permite identificar mucho mejor a los políticos que protagonizaron aquel período y a calibrar adecuadamente el grado de poder que poseían en el círculo provincial y fuera de él . No es éste el lugar para exponer las conclusiones de todos y de cada uno de los trabajos que aquí se han mencionado. Ni hay espacio para ello, ni parecería correcto resumir aquí lo que estos investigadores han publicado ya en una buena parte, o que sin duda publicarán en un futuro inmediato. Ahora bien, sí cabe, aunque sólo sea brevemente, constatar aquí que en Sevilla, la provincia mejor estudiada, tras las dificultades iniciales para la configuración de los dos grandes partidos del "turno", el mecanismo de funcionamiento de la política quedó consolidado a partir de 1881. Sin embargo, no fue hasta comienzos de nuestro siglo cuando las fuerzas políticas se reorganizaron en función de cuatro partidos perfectamente definidos. El partido liberal, que con Pedro Rodriguez de la Borbolla a la cabeza, se convirtió en la fuerza hegemónica. El partido conservador, manejado por la familia Ybarra, que al decir de 
María Sierra, basó su actividad política en el poder económico y en los intereses que necesariamente tenían que defender. En tercer lugar, el partido republicano, cuya jefatura ostentaba José Montes Sierra, banquero y hombre moderado, que se mostró dispuesto a aceptar el juego político de la.Restauración a cambio de un acta de diputado. Y por último, las autodefinidas como "verdaderas derechas", que surgieron por el desencanto de algunos ante lo que entendían como el fracaso de los conservadores.

La desaparición del líder del partido liberal, Rodriguez de la Borbolla, y la fragmentación del conservadurismo entre los distintos grupúsculos en los que fue aglutinándose la derecha sevillana, marcaron la crísis de la Restauración en la provincia. En Huelva, el partido conservador mantuvo su predominio hasta las elecciones de 1923 gracias a la solidez del cacicato de Burgos y Mazo a la debilidad de los liberales, y a la inexistencia de otros grupos con la fuerza suficiente como para restarle apoyos.

La Dictadura de Primo de Rivera no favoreció la unión de esta derecha en Sevilla, que se mostró incapaz de encontrar cauces de unión a pasar de la sensación de terror que la asaltó ante una revolución que se consideraba inminente.

En definitiva, lo que puede afirmarse a la vista de los resultados obtenidos por estas investigaciones hasta el momento presente, es que los partidos del turno, después de una fase de relativa cohesión y solidez, atravesaron por una profunda crísis a partir de la segunda década del siglo XX, debida a su falta de organización, a su escasa implantación y a la, cada vez mayor, desconfianza de la ciudadanía, que rechazaba la debilidad de los programas, las irregularidades de las elecciones y las manipulaciones de que eran objeto por parte de la clase política. La desmovilización del electorado ante la conciencia de que la política era una actividad de un círculo estrecho de iniciados, dio paso a un movimiento tendente a la creación de agrupaciones de elementos de esta sociedad que trataban de defender sus intereses económicos y sociales ante la falta de operatividad, y también de representatividad, de los partidos tradicionales. Eso explica la buena acogida que tuvo entre muchos habitantes de estas provincias el golpe de Estado de 1923.

Pero ni siquiera el advenimiento de la Dictadura sirvió para aglutinar a estas derechas. La proclamación de la República respondía a un fracaso de las derechas más que a un triunfo de las fuerzas progresistas, que sólo tuvieron que prestar su entusiástico apoyo a un régimen que inevitablemente estaba destinado a llenar el hueco dejado por una Restauración fracasada. 
El proyecto no está aún concluido, pero la buena marcha de los trabajos en curso nos permitirá contar en breve con un conocimiento puntual y detallado de los protagonistas de todo este proceso y de los resortes de la política en esta parte de Andalucía durante todo el período de la Restauración, incluidos sus antecedentes y sus consecuentes. 